\title{
Monolithic reconfigurable bandpass filter using single-pole double-throw RF MEMS switches
}

\author{
Jong-Man Kim ${ }^{1 a)}$, Sanghyo Lee ${ }^{2}$, Chang-Wook Baek ${ }^{3}$, \\ Youngwoo Kwon ${ }^{4}$, and Yong-Kweon Kim ${ }^{4}$ \\ ${ }^{1}$ Department of Nanosystem and Nanoprocess Engineering, \\ Pusan National University, \\ Jangjeon 2-dong, Geumjeong-gu, Pusan 609-735, Korea \\ 2 System LSI Division, Samsung Electronics, \\ San \#24, Nongseo-dong, Giheung-gu, Yongin, Gyeonggi-do 446-711, Korea \\ ${ }^{3}$ School of Electrical and Electronics Engineering, Chung-Ang University, \\ 221 Heukseok-dong, Dongjak-gu, Seoul 156-756, Korea \\ ${ }^{4}$ School of Electrical Engineering and Computer Science, \\ Seoul National University, \\ Gwanak P. O. Box 34, Seoul 151-600, Korea \\ a) jongkim@pusan.ac.kr
}

\begin{abstract}
This paper demonstrates a micromachined two-state switchable bandpass filter operating at 2.4 and $5.2 \mathrm{GHz}$. The proposed filter is monolithically composed of two single-crystal silicon (SCS) single-pole double-throw (SPDT) RF MEMS switches and two kinds of two-pole LC-resonators, and it is fabricated by a robust silicon on glass ( $\mathrm{SiOG}$ ) process that combines various micromachining technologies. The insertion losses for each output port of the fabricated SPDT switch was less than $0.15 \mathrm{~dB}$ up to $5 \mathrm{GHz}$. The input to output isolations were higher than $31 \mathrm{~dB}$ for each port. The measured center frequencies of the fabricated reconfigurable filter were 2.9 and $5.4 \mathrm{GHz}$, and the measured passband losses were 2.43 and $5.51 \mathrm{~dB}$ in the low and high frequency bands, respectively.
\end{abstract}

Keywords: MEMS, monolithic reconfigurable filter, SCS, SPDT

Classification: Micro- or nano-electromechanical systems

\section{References}

[1] W. Johler, "RF performance of ultra-miniature high frequency relays," IEEE Holm Conf. Electrical Contacts, pp. 179-189, 2003.

[2] L. P. B. Katehi, J. F. Harvey, and E. Brown, "MEMS and Si micromachined circuits for high frequency applications," IEEE Trans. Microw. Theory Tech., vol. 50, no. 3, pp. 858-866, 2002.

[3] A. Abbaspour-Tamijani, L. Dussopt, and G. M. Rebeiz, "A high performance MEMS miniature tunable bandpass filter," IEEE MTT-S Intl. Microw. Symp. Dig., vol. 3, pp. 1785-1788, 2003. 
[4] A. Pothier, J.-C. Orlianges, G. Zheng, C. Champeaux, A. Catherinot, D. Cros, P. Blondy, and J. Papapolymerou, "Low-loss 2-bit tunable bandpass filter using MEMS dc contact switches," IEEE Trans. Microw. Theory Tech., vol. 53, no. 1, pp. 354-360, 2005.

[5] R. Lempkowski, K. Lian, M. Eliacin, and P. Kulkarni, "A PWBbased MEMS switched filter bank using lumped element embedded passives," The 31 $1^{\text {st }}$ Annual Conf. of the IEEE Industrial Electronics Society (IECON'05), pp. 2331-2334, 2005.

[6] I. C. Reines, C. L. Goldsmith, C. D. Nordquist, C. W. Dyck, G. M. Krauss, T. A. Plut, P. S. Finnegan, F. Austin IV, and C. T. Sullivan, "A low loss RF MEMS Ku-Band integrated switched filter bank," IEEE Microw. Wireless Compon. Lett., vol. 15, no. 2, pp. 74-76, 2005.

[7] J.-M. Kim, J.-H. Park, C.-W. Baek, and Y.-K. Kim, "The SiOG-based single-crystalline silicon (SCS) RF MEMS switch with uniform characteristics," J. Microelectromech. Syst., vol. 13, no. 6, pp. 1036-1042, 2004.

\section{Introduction}

The RF filter is one of the most crucial components in micro- and $\mathrm{mm}$ wave receiving and transmitting systems. Recently, as explosive demands for multi-band RF systems have been increased, the need for a tunable filter has been accordingly emphasizing in order to reduce the complexity and loss of the RF systems as well as to deal with several different frequency bands in a system. Advances in microelectromechanical systems (MEMS) technology have given rise to rapid progress of the tunable filters with superior merits over their electrical counterparts in terms of loss, linearity, power consumption, and compactness $[1,2]$. Generally, the frequency tuning methods of the MEMS-based filters can be classified into two methods. One is to tune the frequencies by changing the inductance and capacitance values of the resonators using various micromachined lumped tuning elements [3, 4]. Diverse combinations of the tuning elements enable the reconfigurable compact filter to be constructed monolithically. However, in these kinds of filters, the filter design can be somewhat fastidious since so many tuning elements are often required to get wide tuning ranges. Moreover, the use of several individual tuning elements in a filter can bring out difficulties in fine frequency tuning owing to uncertainness about the operation of each tuning element. The other method is to select the fixed resonators having specific center frequencies due to $\mathrm{ON} / \mathrm{OFF}$ digital operations of the switching elements. In this method, the complexities of filter design can be greatly alleviated, and a fine frequency tuning with a broad tuning range is achievable because the center frequencies of the filters are determined only by choosing the pre-fixed filter banks that have specific frequencies $[5,6]$. Nevertheless, there is still a cumbersome process to externally assemble the separately fabricated switches and filter elements by means of wiring or a solder reflow process, resulting in an increase of the filter size and parasitic components. In this paper, we present a two-state reconfigurable bandpass filter, which is monolithically composed 
of two SCS SPDT switches and two LC-resonators fabricated on the same substrate by a batch process. Therefore, external integration and additional wiring processes are not required, so fabrication complexities and parasitic problems can be efficiently reduced. Another outstanding merit of the proposed filter is the implementation of mechanically stable SCS MEMS switches to select the filter banks. The utilized switches are free from structural deformations thanks to the robust nature of the SCS. Therefore, fabrication and performance yields can be improved compared to metallic switches.

\section{Design}

All the components of the proposed reconfigurable filter were designed using a circuit simulator, an advanced design system (ADS), and a full-wave electromagnetic (EM) simulator, IE3D. The two RF signal paths of the SCS SPDT switch branch out symmetrically from one input port and two identical direct-contact type SCS switches are utilized to control the RF paths. The two switches are independently actuated by individually applied bias voltages to alternatively select the RF path. All ground planes are connected by metallic structures in order to suppress the parasitic modes on discontinuous areas of the CPW line. The ground connection line is separated from the bottom signal lines by an intermediate silicon dioxide $\left(\mathrm{SiO}_{2}\right)$ insulator layer having a dielectric constant of 4.5. The thickness of the oxide layer was designed to be $1.5 \mu \mathrm{m}$ by simulation using the ADS to minimize both the RF losses and the fabrication difficulties. A plane view of the designed reconfigurable filter with the simplified equivalent circuit of the resonator is illustrated in Fig. 1. The proposed reconfigurable filter consists of two different two-pole resonators having center frequencies of 2.4 and $5.2 \mathrm{GHz}$, and two SPDT switches to control the RF signal flows. Each LC-resonator is composed of spiral inductors and metal-insulator-metal (MIM) capacitors having

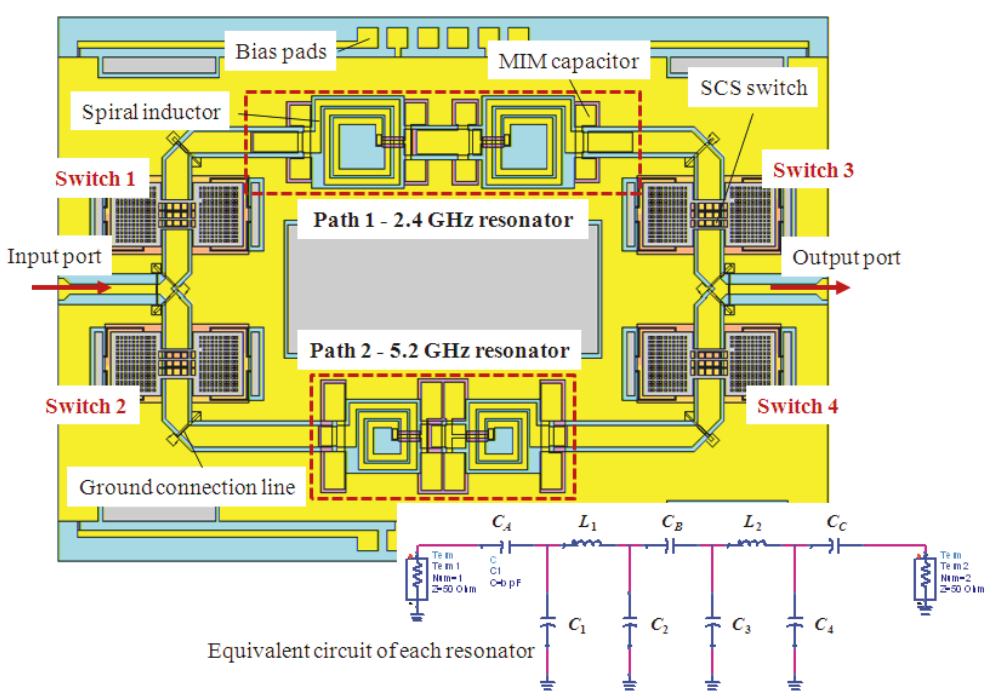


fixed inductance and capacitance values. Two SPDT switches are connected to each resonator and play the role of the input and output ports of the filter. Consequently, a total of 4 single SCS switches were utilized in the filter and the filter operations are entirely achieved by ON/OFF operations of the implemented switches. As shown in Fig. 1, when switches 1 and 3 are actuated together by the bias voltage, the applied input RF signal flows to the output port through path 1 , so the center frequency of the filter is $2.4 \mathrm{GHz}$. In this case, path 2 still maintains its deactivated state. On the other hand, when switches 2 and 4 are simultaneously turned on instead of switches 1 and 3, the center frequency of the filter moves to $5.2 \mathrm{GHz}$. This filter topology can provide a precise control of the filter responses because each fixed filter bank is only selected by operations of the implemented SPDT switches. Moreover, mechanically robust SCS switches can provide stable actuations, resulting in reliable filter operations. Compact size and a simple configuration of the filter are also possible since the switches and the resonators are fabricated on a same substrate by a batch process. The HFSS simulations for the designed filter were performed and compared with the simulation results of each resonator without combining them using the SPDT switches in order to show the influences of the switches on the RF responses. In this case, the contact resistance of $2 \Omega$ was considered at the switch location in the activated path. As demonstrated by the simulation results, significant changes in the filters' own RF responses were not observed except for a slight increase of passband losses due to the contact resistances of the switches.

\section{Fabrication}

The proposed reconfigurable filter is fully fabricated by the SiOG process, which is very useful for making deform-free structures based on the silicon DRIE process and the silicon/glass anodic bonding technique [7]. The scanning electron microscope (SEM) images for the fabricated reconfigurable filter are shown in Fig. 2 (a). Two SPDT switches were fabricated with no structural deformations due to the mechanical robustness of the SCS. In order to more precisely observe the fabricated structures, we measured the 3D profile

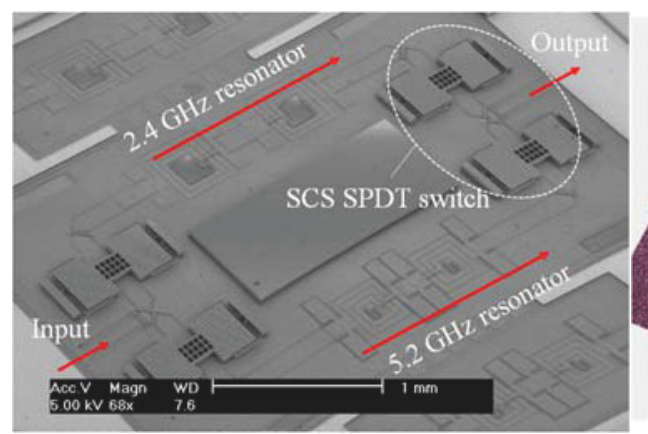

(a)
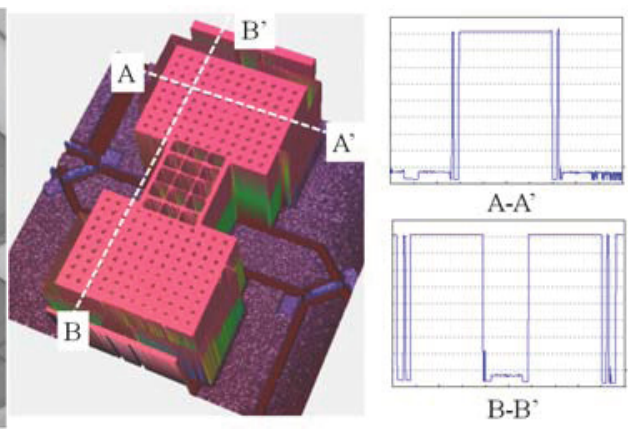

(b)

Fig. 2. Fabrication results. (a) SEM pictures for the fabricated reconfigurable filter, and (b) 3-D profile with cross-sectional views of the SPST switch. 
with cross-sectional views of the SPST switch as shown in Fig. 2 (b).

The fabricated moving plates and springs of the switch maintained the same height along the scanned lines, and this clearly reveals that no structural deformations occurred.

\section{Measurement}

\subsection{SPDT switch}

The proposed SCS SPDT switch has one input port and two RF paths, which are symmetric with respect to the input port, and it is implemented using two SPST switches that are individually actuated with separately applied bias voltages to select the RF path. The RF characteristics of the fabricated SPDT switch were evaluated by measuring the S-parameters using an 8510C network analyzer with a frequency range of DC to $10 \mathrm{GHz}$ after a short-openload-thru (SOLT) calibration process. In all cases, a bias voltage of $30 \mathrm{~V}$ was applied to actuate the switches. The insertion and return losses were characterized by measuring $\mathrm{S}_{21}$ and $\mathrm{S}_{11}$ for the activated path, respectively. The input to output isolations were determined by measuring $S_{21}$ of the deactivated path as the other path was activated. The measured RF performances for each path of the SPDT switch with the HFSS simulation results are shown in Fig. 3 (a). The measured insertion losses were less than 0.15 and $0.13 \mathrm{~dB}$ up to $5 \mathrm{GHz}$ for paths 1 and 2, respectively. As one path was activated, the measured isolation characteristics between the input and output ports of the deactivated path were higher than 31 and $33 \mathrm{~dB}$ up to $5 \mathrm{GHz}$ for paths 1 and 2 , respectively. The measured RF performances for the two paths were very close each other because the two RF paths that branched from the input port are symmetric with respect to the input port.

\subsection{Reconfigurable filter using the SPDT switches}

Tunability of the proposed filter can be achieved by choosing the resonators having the different center frequencies of 2.4 and $5.2 \mathrm{GHz}$. As mentioned above, the proposed filter is made monolithically by the combinations of the

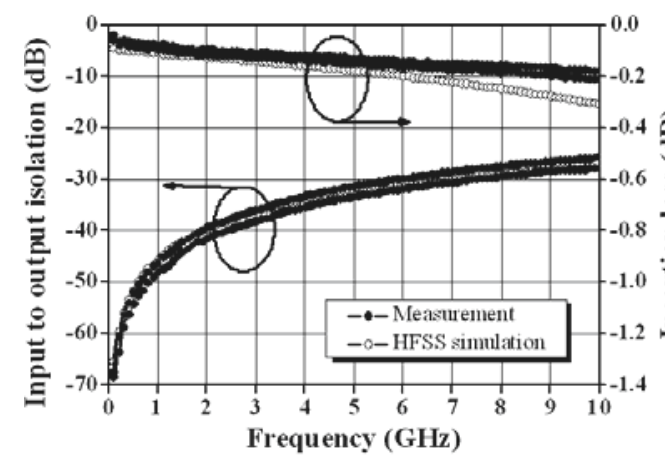

(a)

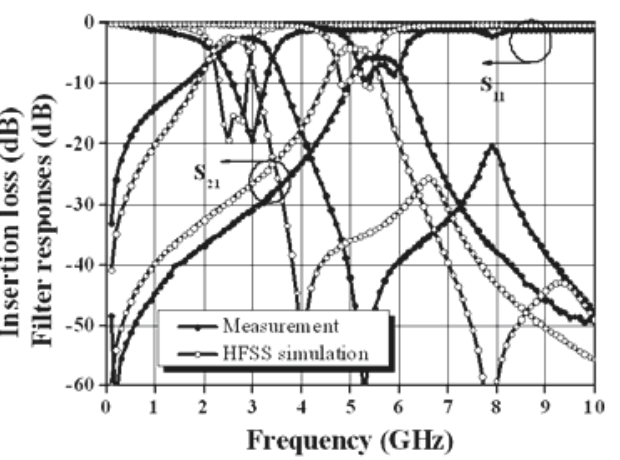

(b)

Fig. 3. Measured RF responses with the HFSS simulation results. (a) SPDT switch, and (b) reconfigurable filter using the SPDT switches. 
switches and the resonators. Therefore, the losses generated by the switch contact resistance during actuations were added to the passband loss of the resonators. However, we only measured the filter responses because the simulations demonstrated that adding the switch had little influence on the frequency responses of the filter. The filter responses were also measured using the network analyzer with a frequency range of DC to $10 \mathrm{GHz}$. The measured $\mathrm{RF}$ responses of the fabricated reconfigurable filter with the HFSS simulation results are shown in Fig. 3 (b). In the initial state with no actuations of the switches, there were no filter responses since the filter is initially regarded as an open circuit. As switches 1 and 3 were actuated together with the applied voltage of $30 \mathrm{~V}$, resonator 1 (the $2.4 \mathrm{GHz}$ filter bank) was selected and switches 2 and 4 still maintained their initial OFF states. In this case, the center frequency of the filter was measured to be $2.9 \mathrm{GHz}$ with a passband insertion loss of $2.43 \mathrm{~dB}$. On the other hand, as path 2 was activated by the simultaneous actuations of switches 2 and 4, the center frequency was shifted to the high frequency band. In this case, the measured center frequency and the insertion loss were $5.4 \mathrm{GHz}$ and $5.51 \mathrm{~dB}$, respectively. The measured center frequencies were slightly shifted compared to the target frequencies. It is believed that these frequency errors were caused by uncertainness of the dielectric constant of the TEOS oxide layer that was used for the MIM capacitors. However, these disagreements between the targeted and the measured frequency may be sufficiently resolved by confirming the exact dielectric constant of the silicon dioxide layer.

\section{Conclusion}

In this paper, the micromachined monolithic reconfigurable filter using two SCS SPDT switches and two resonators having the different frequencies of 2.4 and $5.2 \mathrm{GHz}$ was successfully demonstrated. The monolithic combination of the switches and the resonators resulted in structural simplicity of the filter with little parasitic effects. Because the frequency tuning of the filter could be achieved by the actuations of the implemented switches, the design procedures became easier and fine tuning was also possible. With the aid of the robust SiOG process, a mechanically stable filter and switches with uniform performance could be fabricated. Each RF path of the fabricated SPDT switch showed insertion losses lower than 0.15 and $0.13 \mathrm{~dB}$ from DC to $5 \mathrm{GHz}$ for output ports 1 and 2, respectively. The measured isolation characteristics between the input and the deactivated path were higher than 31 and $33 \mathrm{~dB}$ up to $5 \mathrm{GHz}$ for paths 1 and 2, respectively. The measured center frequencies and passband losses of the fabricated monolithic switchable filter were $2.9 \mathrm{GHz}$ and $2.43 \mathrm{~dB}$, respectively for the low frequency band, and $5.4 \mathrm{GHz}$ and $5.51 \mathrm{~dB}$, respectively for the high frequency band. All the measured results were also well-matched with the simulation results. These results clearly indicate that the monolithic reconfigurable filter proposed in this paper has great potential for application in multi-band RF systems. 


\section{Acknowledgement}

This work was supported by Korea Ministry of Science and Technology through the Creative Research Initiative Program. 